\title{
A Large-Scale Chinese Short-Text Conversation Dataset
}

\author{
Yida Wang ${ }^{1}$, Pei Ke ${ }^{2}$, Yinhe Zheng ${ }^{2,3}$, Kaili Huang ${ }^{2}$, Yong Jiang ${ }^{1}$, Xiaoyan \\ $\mathrm{Zhu}^{2}$, and Minlie Huang ${ }^{2}$ * \\ 1 Tsinghua Shenzhen International Graduate School, Tsinghua University, China \\ 2 Institute for Artifical Intelligence, State Key Lab of Intelligent Technology and \\ Systems. Beijing National Research Center for Information Science and Technology. \\ Department of Computer Science and Technology, Tsinghua University, Beijing, \\ China. \\ 3 Samsung Research China - Beijing (SRC-B), Beijing, China. \\ \{wangyd18,kp17,hkl16\}@mails.tsinghua.edu.cn, yh.zheng@samsung.com, \\ jiangy@sz.tsinghua.edu.cn, \{zxy-dcs, aihuang\}@tsinghua.edu.cn
}

\begin{abstract}
The advancements of neural dialogue generation models show promising results on modeling short-text conversations. However, training such models usually needs a large-scale high-quality dialogue corpus, which is hard to access. In this paper, we present a large-scale cleaned Chinese conversation dataset $L C C C$, which contains a base version (6.8 million dialogues) and a large version (12.0 million dialogues). The quality of our dataset is ensured by a rigorous data cleaning pipeline, which is built based on a set of rules and a classifier that is trained on manually annotated $110 \mathrm{~K}$ dialogue pairs. We also release pre-training dialogue models which are trained on LCCC-base and LCCC-large respectively. The cleaned dataset and the pre-training models will facilitate the research of short-text conversation modeling. All the models and datasets are available at https://github.com/thu-coai/CDial-GPT.
\end{abstract}

Keywords: Deep learning · Dialogue generation · Dataset · Pre-training.

\section{Introduction}

The success of pre-training models has greatly advanced the research of natural language processing [21. While BERT [5] promotes various natural language understanding tasks, GPT 23] demonstrates state-of-the-art performance on natural language generation. Recently, pre-training models have been applied to dialogue generation tasks and achieved state-of-the art results [3831].

In addition to the effective Transformer-based model structures, dialogue corpora also play an important role in the success of open-domain dialogue generation models. Existing work has adopted massive English dialogue corpora from Twitter 24, Reddit [19, OpenSubtitles [14] and other public resources,

\footnotetext{
* Corresponding author
} 
which equip the pre-training models with the ability to respond to human in open domains.

However, since there are few large-scale Chinese dialogue corpora, the development of the pre-training models for dialogue generation in Chinese has been hindered. We argue that it's essential to construct high-quality large-scale Chinese dialogue corpora which can further promote pre-training models in Chinese. The main challenge we need to face is the quality control of dialogue data. On the one hand, we cannot rely on crowd-sourcing services because existing work has shown that the amount of data used in the pre-training models is quite large 23 . On the other hand, if we mainly acquire data from public resources such as social media (Weibo, Twitter, Reddit, etc.), the quality of the data will be hard to ensure. As a matter of fact, online social media contains many negative behaviors, including toxic comments (i.e. comments that are rude, disrespectful or otherwise likely to make someone leave a discussion) 4 , threats, insults, identity hates, obscene contents, and many more. These factors can remarkably degrade the generation ability of dialogue generation models, and lead to serious unexpected behaviors, which substantially limit the practical use of dialogue models.

In this work, we construct a large-scale cleaned Chinese conversation dataset called LCCC, which contains two versions, LCCC-base and LCCC-large. LCCCbase is filtered from 79 million conversations crawled from Weibo, while LCCClarge is filtered from the combination of Weibo data and other sources of Chinese corpora. A two-phase pipeline is designed to clean this corpus. Specifically, the first phase utilizes a set of heuristic rules to filter out dialogues with inappropriate content, and the second phase employs several classifiers that are trained on manually labeled data to filter dialogues further. Then we present pre-training models for dialogue generation, which are first pre-trained on a Chinese novel dataset and then post-trained on LCCC. All the pre-trained models and the datasets are released to facilitate future research.

Our contributions can be summarized as below:

- We build a large-scale cleaned Chinese conversation dataset called LCCC. It can serve as a benchmark for the study of open-domain conversation generation in Chinese.

- We present pre-training models for Chinese dialogue generation. Moreover, we conduct experiments to show its performance on Chinese dialogue generation. Both the models and the data are released for public use.

\section{Related work}

Datasets We make a brief overview of datasets available for data-driven conversation systems, most of which are constructed based on public resources or crowd-sourcing.

\footnotetext{
${ }^{4}$ https://www.kaggle.com/c/jigsaw-toxic-comment-classification-challenge
} 
As an important public resource of dialogue corpora, movie scripts such as OpenSubtitles have been used to construct dialogue datasets [14|26]. The dialogue content usually depends on the scenes of movies. Another public resource is social media, where Twitter [11|30, Reddit 19], Weibo [33|28, and technical forums [17 have been adopted to build open-domain dialogue datasets. These datasets crawled from public resources are usually at a large scale because the corpora of public resources is abundant. However, they also contain much noise which need to be carefully cleaned.

Other researchers resort to construct high-quality dialogue datesets by crowdsourcing. These datasets are built for advanced dialogue tasks, such as wizard of wikipedia (WoW) [6] / document grounded conversations (DoG) [0] for knowledge grounded dialogue generation, PERSONA-CHAT [37] for persona enhanced dialogue generation, and DailyDialog [13] for emotional conversation generation. These datasets are commonly cleaner than those acquired from public resources, but the amount of these datasets is rather small.

Pre-training Models on Dialogue Generation Since GPT [22] achieves state-of-the-art performance on various text generation tasks, recent work has applied pre-training models to dialogue generation. DialoGPT [38] presents an English open-domain pre-training model which post-trains GPT-2 [23] on 147M Reddit conversations. Meena [1] trains an Evolved Transformer [29] with 2.6B parameters on a massive English social media conversation dataset, which contains 40B words. [12] investigates dialogue generation by fine-tuning a Chinese GPT on some small dialogue datasets, where the Chinese GPT is pre-trained on a Chinese corpus mixed with Chinese Wikipedia2 (1.7B words) and Chinese News (9.2B words).

\section{Datasets}

We crawled 79M conversations from Weibo. First of all, through a rigorous cleaning process, a cleaned Weibo dataset (LCCC-base) was constructed. Then, the $79 \mathrm{M}$ conversations were mixed with the several public Chinese conversation datasets, and a larger Chinese conversation dataset (LCCC-large) was obtained via more relaxed cleaning conditions. The cleaning process includes rule-based and classifier-based filtering.

\subsection{Data Collection}

LCCC-base A two-phase data collection scheme is used to construct our raw dialogues. At the first phase, a set of seed users were collected. Specifically, we manually selected a batch of Weibo accounts who follow professional mass media dedicating to publish news. We then regarded the users who post comments under these news as "high-quality" users, since robot accounts usually do not pay much attention to these daily news. 


\begin{tabular}{|c|c|}
\hline$\overline{\text { Type }}$ & Case \\
\hline Platform tag & |回复@精灵小宝贝 : 我也失眠了 \\
\hline Advertisement & @张伟丽 MMA 前来为DW持妆粉底液实力证言! \\
\hline Generic form & 我也是 我也是 我也是 啊啊 \\
\hline Dirty word & 被小婊砸作的现在满身负能量 \\
\hline Special word & 可以试试左氧氟沙星 \\
\hline Name & 陈绍龙、你无朋友架啦 \\
\hline Symbol & $\#\left({ }^{\circ}-{ }^{\circ}\right) \#$ \\
\hline Platform sign & 文科574报哪里好?最好有师范英语【微信】 \\
\hline Not fluent & Q: 昨晚失眠了 A: 发恶梦扎醒,又发我最怕个蒋尸梦! \\
\hline Incomplete information & Q: 江南小镇很美呀 A:印象 \\
\hline Time-sensitive & Q: 感觉没啥电视好看了 A: 琅哪榜, 就要大结局了 \\
\hline External noun & Q: 假期要过了 A: 春节一过,好想立刻回到岛城 \\
\hline Irrelevant pairs & Q: 差点吧洗面奶当牙膏 A: 绿色是今年的流行色 \\
\hline
\end{tabular}

Table 1. Cases of noise in the cleaning process.

At the second phase, we collected dialogues from these seed users. Specifically, the Weibo posts from these users are collected along with the following comments, which are organized in tree structures. Note that any path from a root to a leaf can be regarded as a conversation session. We reconstructed these sessions using a Depth First Search process, and 79 million sessions of raw conversations were constructed. We then constructed a cleaned Weibo dataset using the cleaning method described in Section 3.2 .

LCCC-large We collected corpora from multiple open-source repositories, including Chinese Chatterbot Corpus 5, PTT Gossiping Corpus 6, Subtitle Corpus and Xiaohuangji Corpus 7. These datasets, together with Qingyun Corpus and Tieba Corpus, are cleaned and processed to be single-turn conversational data 8. Besides, we collected multi-turn conversational data including Douban Conversation Corpus 9, E-commerical Conversation Corpus 10 and a Chinese chat corpus 11 .

We then mixed these datasets with the $79 \mathrm{M}$ conversations. Using the same cleaning process, but by relaxing the threshold of the classifier described below, we obtained a larger version of our dataset (LCCC-large).

\subsection{Cleaning Process}

Rule-based Noise Filtering We filter out many types of noise via rules including: (1) delete the platform tag in the dialogues, such as "Reply to @***",

\footnotetext{
${ }^{5}$ https://github.com/gunthercox/chatterbot-corpus

${ }^{6}$ https://github.com/zake7749/Gossiping-Chinese-Corpus

7 https://github.com/skdjlla/dgk_lost_conv

${ }^{8}$ https://github.com/codemayq/chinese_chatbot_corpus

${ }^{9}$ https://github.com/MarkWuNLP/MultiTurnResponseSelection

${ }^{10}$ https://github.com/cooelf/DeepUtteranceAggregation

${ }^{11}$ https://github.com/yangjianxin1/GPT2-chitchat
} 
"[dog]"; (2) remove URL strings from the text; (3) split conversations with more than 30 turns into multiple conversations less than 30 turns [28; (4) only keep one copy of the phrases or words that are repeated more than 6 times in one sentence; (5) remove the dialogues if the response is too long or too short; (6) remove the dialogues if the response is identified as an advertisement by the method in 33 ; (7) remove the dialogues if $90 \%$ of tri-grams in the response are high-frequency tri-grams [38; (8) remove the dialogues if the response has some specific forms of generic responses; (9) remove the dialogues in which the response is the same as the post.

We also construct blacklists containing the following noise: (1) dirty words, sensitive words, and dialect; (2) special topics words such as levofloxacin; (3) name, appellation and unknown abbreviation; (4) special symbols and emoji; (5) platform signs such as ads, pictures, and videos related words. The dialogue will be removed if it contains words that appear in the blacklist. Some cases are shown in Tabel 1 (top).

Classifier-based Filtering In addition to the rule-based method, we also introduce classifier-based filtering. Many types of noise in terms of semantics and grammar, and some context-dependent conversation, 12 are hard to be filtered with rules. So we built two BERT classifiers for more elaborate filtering. We evaluated precision, recall, and F-score with different confidence scores to choose the best confidence threshold.

The first BERT classifier was trained on manually labeled 100,000 conversations. A dialogue is labeled noisy if it has the above noise or following noise: (1) The response is not fluent or there are serious typos in the sentence, (2) The information of the response is incomplete; (3) The topic of dialogue is timesensitive, (4) Festivals, places, gender and time which are not mentioned in the post appear in the response (5) The post and the response are irrelevant. Some cases are shown in Tabel1(bottom). The classification accuracy reaches $73.76 \%$ on the test set.

In social media, many conversations inevitably depend on external contexts beyond the text 27/33] making them hard to understand 9]. To alleviate this problem, the second BERT classifier was trained on a manually labeled dataset containing 10,000 utterances. The classification accuracy reaches $77.60 \%$ on the test set.

\subsection{Statistics and Results}

The statistics about the dataset are shown in Table 2 The Avg.words means the average number of words per utterance, and text is tokenized by Jieba 13 . We also estimated the noise level in the STC dataset via our blacklist. Results show that $60 \%$ of conversations in STC contain dirty words, sensitive words, special symbols, etc. The model trained on STC generates five times more blacklist

12 The understanding of the first post depends on other context beyond the post.

13 https://github.com/fxsjy/jieba 


\begin{tabular}{l|cc|cc}
\hline & Single-Turn & Multi-Turn & Single-Turn Multi-Turn \\
\hline Raw dialogs & $52,708,955$ & $26,749,365$ & $63,251,887$ & $28,189,952$ \\
Cleaned dialogs & $3,354,277$ & $3,466,278$ & $7,273,804$ & $4,733,955$ \\
Utterances & $6,708,554$ & $13,365,268$ & $14,547,608$ & $18,341,167$ \\
Characters & $68,559,727$ & $163,690,614$ & $162,301,556$ & $217,776,649$ \\
Vocabulary size & 372,063 & 666,931 & 662,514 & 690,027 \\
Avg. words & 6.79 & 8.32 & 7.45 & 8.14 \\
Avg. turns & 2 & 3.86 & 2 & 3.87 \\
\hline
\end{tabular}

Table 2. Statistics of LCCC-base (left) and LCCC-large (right).

\begin{tabular}{|c|c|c|c|}
\hline Dataset & Corpus Statistics & Source Topic & Corpus Features \\
\hline DuConv 35. & $\begin{array}{l}29,858 \text { dialogs } \\
9.1 \text { turns per dialog } \\
10.6 \text { words per turn }\end{array}$ & $\begin{array}{ll}\text { Crowd } & \text { Films and } \\
\text { source } & \text { film stars }\end{array}$ & $\begin{array}{l}\text { Knowledge-grounded/ } \\
\text { Proactivity modeling }\end{array}$ \\
\hline Douban 36 & $\begin{array}{l}0.5 \mathrm{M} \text { dialogs } \\
7.69 \text { turns per dialog } \\
18.56 \text { words per turn }\end{array}$ & an Ope & $0.5 \mathrm{~N}$ \\
\hline $\begin{array}{l}\text { Persona- } \\
\text { Dialog } 39\end{array}$ & $\begin{array}{l}\text { 20.83M dialogs } \\
56.26 \mathrm{M} \text { utterances } \\
\text { 8.47M user profiles }\end{array}$ & Weibo Open topics & $\begin{array}{l}\text { Personalization,rich } \\
\text { user profiles }\end{array}$ \\
\hline STC 28 & $\begin{array}{l}\text { 4.4M pairs } \\
219,905 \text { posts } \\
4.3 \mathrm{M} \text { responses }\end{array}$ & Weibo Open topics & $\begin{array}{l}\text { One post multiple } \\
\text { responses }\end{array}$ \\
\hline $\mathrm{LCCC}_{\text {base }}$ & $\begin{array}{l}6.8 \mathrm{M} \text { dialogs } \\
2.95 \text { turns per dialog } \\
20 \mathrm{M} \text { utterances }\end{array}$ & Weibo Open topics & $\begin{array}{l}\text { Extremely Strict cleaning } \\
\text { process }\end{array}$ \\
\hline $\mathrm{LCCC}_{\text {large }}$ & $\begin{array}{l}\text { 12M dialogs } \\
2.74 \text { turns per dialog } \\
33 \mathrm{M} \text { utterances }\end{array}$ & Mixup Open topics & Strict cleaning process \\
\hline
\end{tabular}

Table 3. Comparison between existing Chinese conversation datasets and LCCC.

words than that trained on LCCC. In Table 3, a clear comparison between the existing Chinese dialogue dataset and our dataset is presented.

\section{Models}

Architectures The model architecture used in this paper is adopted from GPT 22 which is based on transformer [32] . The transformer decoder consists of multiple masked multi-head self-attention blocks. In each time step, the selfattention mechanism can only observe the information on the left.

Given a golden response $y=\left(y^{1}, \ldots, y^{L}\right)$ and history utterances of a conversation $U=\left\{u_{0}, \ldots, u_{n}\right\}$ where each utterance $u_{i}=\left(u_{i}^{1}, \ldots, u_{i}^{L_{i}}\right)$ consists of $L_{i}$ words, our goal is train a generation model via maximum likelihood estimation (MLE) $P(y \mid U)=\prod_{j=1}^{L} P\left(y^{j} \mid y^{1}, \ldots, y^{j-1}, U\right)$ to generate $u_{n+1}^{j}$ given $U$ with generated $u_{n+1}^{1}, \ldots, u_{n+1}^{j-1}$ until the whole response is generated with one termination symbol. 
Input Representation We concatenate all history utterances into one sequence as a long text described in [34. The input of the model is the sum of word embedding, speaker embedding, and position embedding. The word embedding and position embedding are learned during the pre-training phase, and speaker embedding is learned during the post-training or fine-tuning phase. The speaker embedding is used to indicate the different speakers, and we use the speaker symbol as the separation tokens. Following BERT [5], we also add a $[C L S]$ to the start of the sequence and use $[S E P]$ as the end-of-sequence token.

Training Following the work of DialoGPT [38, our models are post-trained based on a pre-trained Chinese GPT model $\left(\mathrm{GPT}_{\text {Novel }}\right)$ on the conversation dataset we collected. For multi-turn dialogue instances, following DialoGPT, We take every sentence in the dialogue from the second sentence to the last sentence as the response of history sentences. We trained several models on the LCCC-base and LCCC-large, respectively (LCCC-base is cleaner), which are summarized as follows:

- GPT $_{\text {Novel }}$ is a 12-layer GPT which is pre-trained for 70 epochs on a Chinese novel dataset consisting of a variety of genres (Comedy, Romance, Mystery) with about 0.5 billion tokens.

- CDialGPT $_{\mathrm{LCCC}-\text { base }}$ is a 12-layer GPT which is pre-trained for 70 epochs on the Chinese novel dataset and post-trained for 30 epochs on LCCC-base.

- CDialGPT2 $2_{\mathrm{LCCC}-\text { base }}$ is a 12 -layer GPT2 which is pre-trained for 70 epochs on the Chinese novel dataset and post-trained for 30 epochs on LCCC-base.

- $\mathrm{CDialGPT}_{\mathrm{LCCC}-\text { large }}$ is a 12-layer GPT which is pre-trained for 70 epochs on the Chinese novel dataset and post-trained for 30 epochs on LCCC-large.

All models were optimized by the AdamW [16] optimizer and the Noam 22 . learning rate decay method. The layers of all our models are set to 12 , and the number of heads is set to 12 . The dimension of word embedding is set to 768 , and the dimension of position embedding is set to 513 . The number of the warmup epoch was set to 1 , and the maximum learning rate was $6.25 \mathrm{e}-5$. The batch size was set to 8 , and the number of gradient accumulation was set to 64. All the models trained with a vocabulary of 13,088 Chinese characters on four RTX 2080ti GPUs. Our implementation is based on the open-source code of Transformers 14 and TransferTransfo 15

\section{Experiments}

\subsection{Fine-tuning Settings and Implementation Details}

To evaluate the models, we fine-tune all our models mentioned in Section 4 on the STC dataset. The STC dataset contains $4.4 \mathrm{M}$ conversation pairs. We randomly split the dataset into train, validation, and test sets. The validation set and the test set have 20,000 conversation pairs each without any intersection.

${ }^{14}$ https://github.com/huggingface/transformers

15 https://github.com/huggingface/transfer-learning-conv-ai 
(a) An interactive example of multiturn dialogue generation.

\begin{tabular}{l|l}
\hline Role & Response \\
\hline User & 别爱我没结果 \\
Bot & 没结果的爱是多么的痛。 \\
User & 但是爱过就可以了 \\
Bot & 有时候, 爱情就是一场游戏。 \\
User & 输的总是我 \\
Bot & 我爱你, 就像我爱你一样。 \\
User & 你可拉到吧 \\
Bot & 你可拉到吧。 \\
User & 别学我说话 \\
Bot & 我说话就是你不行。 \\
\hline
\end{tabular}

(b) An example of multi-turn selfplaying dialogue generation.

\begin{tabular}{|c|c|}
\hline$\overline{\text { Role }}$ & Response \\
\hline$\overline{\text { User }}$ & 生命的意义是什公 \\
\hline Bot1 & $\begin{array}{l}\text { 生命的意义是过得比你想象 } \\
\text { 的要好 }\end{array}$ \\
\hline Bot2 & 也许吧 \\
\hline Bot1 & $\begin{array}{l}\text { 嗯, 生命的意义是过得比你 } \\
\text { 你想象的要好 }\end{array}$ \\
\hline Bot2 & 说的好 \\
\hline Bot1 & 好什么 \\
\hline Bot2 & 过得比你想象的好 \\
\hline Bot1 & 好吧 \\
\hline
\end{tabular}

Table 4. Two generated examples. In the right table, the user prompt is the initial input (the first row). Both of the bots are the same models (CDialGPT $\left.\mathrm{LCCC}_{\text {-large }}\right)$.

Baselines First we choose the only Chinese dialogue pre-training model GPT2chitchat 16 as one baseline which is pre-trained on 500k Chinese conversations based on the GPT2 23 model. Then we choose the the vanilla transformer 32 as one of the baselines. This model is widely applied in machine translation and conversation generation [19:37. We also evaluate the classical sequence-tosequence 4 431] model with attention mechanism 2] (Attn-Seq2Seq) which has been widely used in conversation generation.

Training Details The GPT $_{\text {Novel }}$ is fine-tuned up to 30 epochs. All the other models are fine-tuned up to 10 epochs with the same batch size and number of gradient accumulation. The other hyper-parameters are also kept the same as the post-training process. The transformer and Attn-Seq2Seq are trained until convergence on STC without pre-training. For a fair comparison, Attn-Seq2Seq consists of 6 layers of GRU with Bahdanau [2] attention mechanism, and the dimension of hidden states is set to 768 . The layers of the transformer are also set to 6 . Thus, these two models have comparable parameters with pre-training models. We set the parameters of GPT2-chitchat to be the same with the original repository. For all the models, we linearly decay the learning rate from $6.25 \mathrm{e}-5$ to 0 using the AdamW optimizer.

Case Study Some cases generated by CDialGPT $\mathrm{LCCC}_{\mathrm{L}-\text { large }}$ which is fine-tuned on STC are provided in Table 5. These samples suggest that the model has the ability to generate informative responses. Following the work of DialoGPT [38, we provide an interactive dialogue sample and a self-playing dialogue sample in Tabel 3(a) and Tabel $3(\mathrm{~b})$. All the above samples are decoded by top-p sampling [8] with temperature 0.7 .

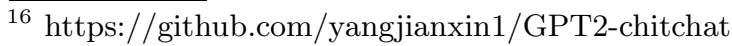




\begin{tabular}{ll}
\hline Post & Response \\
作为一名伟大的吃货你必须知道的事情。 & 大半夜的不睡觉, 你是在诱惑我么? \\
惊现特种喵! 这身手。可以维护世界和平了吧。 & 这猫太有才了, 这猫很有想象力啊! \\
超萌的考拉。不怕痒, 挠吧。 & 我的小心肚要跳出来了 \\
你觉得最好看的英剧Top3是什么? & 我的英剧top3是《老婆婆》 \\
无论身在何方, 我心里满满都是你! & 此时此刻, 我只想说一句: 好可爱! \\
安东尼刷屏, 可惜小手太小, 就是投不进啊 & 这小子太牛了, 真想给他一拳。 \\
披萨饼盒瞬间变笔记本支架, 这个太酷了! & 这个真的可以有。但是不是很实用 \\
无论你多么讨厌你的学校, 当你离开久了, 你 & 还是学校好, 没有负担, 没有压力 \\
还是会想念它。 & \\
\hline
\end{tabular}

Table 5. Cases generated by CDialGPT $\mathrm{LCCC}_{\mathrm{C}-\text { large }}$ fine-tuned on STC.

\subsection{Evaluation}

\begin{tabular}{lccccccccc}
\hline Models & Size & PPL & BLEU-2 & BLEU-4 & Dist-1 & Dist-2 & \multicolumn{2}{c}{$\begin{array}{c}\text { Greedy } \\
\text { Matching }\end{array}$} & $\begin{array}{c}\text { Embedding } \\
\text { Average }\end{array}$ \\
\hline Attn - Seq2Seq & $73 M$ & 34.2 & 3.93 & 0.90 & 0.0085 & 0.1191 & 0.6584 & 0.8338 \\
Transformer & $113 M$ & 22.10 & $\mathbf{6 . 7 2}$ & 3.14 & 0.0088 & 0.1397 & 0.6606 & 0.8355 \\
GPT2 - chitchat & $88 M$ & - & 2.28 & 0.54 & $\mathbf{0 . 0 1 0 3}$ & $\mathbf{0 . 1 6 2 5}$ & 0.6154 & 0.7894 \\
GPT $_{\text {Novel }}$ & $104 M$ & 21.27 & 5.96 & 2.71 & 0.0080 & 0.1172 & 0.6612 & 0.8334 \\
CDialGPT $_{\text {LCCC-base }}$ & $104 M$ & 18.38 & 6.48 & 3.08 & 0.0083 & 0.1268 & 0.6621 & 0.8354 \\
CDialGPT2 $_{\text {LCCC-base }}$ & $104 M$ & 22.76 & 5.69 & 2.50 & 0.0077 & 0.1087 & 0.6624 & 0.8346 \\
CDialGPT $_{\text {LCCC-large }}$ & $104 M$ & $\mathbf{1 8 . 2 3}$ & 6.63 & $\mathbf{3 . 2 0}$ & 0.0083 & 0.1271 & $\mathbf{0 . 6 6 2 5}$ & $\mathbf{0 . 8 3 6 3}$ \\
\hline
\end{tabular}

Table 6. Automatic results.

Automatic Metric We first employed BLEU [20 and distinct n-grams 10] as our automatic metrics. Since BLEU cannot perfectly reflect the quality of generated results [15], we adopted Greedy Matching 25] to evaluate the relevance between posts and generated responses at the word level and Embedding Average [15] at the sentence level. We also present the perplexity [18] of different models except GPT2-chichat which has different vocabularies with others.

As shown in Table 6, the models trained on LCCC achieves the best perplexity (PPL) but worse BLEU-2 scores than Transformer, which is consistent with previous work [3812. 1] shows a strong correlation between perplexity and human evaluation, so our model has competitive performance. The models trained on LCCC also outperforms others in Greedy Matching and Embedding Average. The GPT2-chitchat reaches the highest distinct scores but poor generation quality where we attribute it to the small scale of the model.

Human Evaluation We also employed human evaluation to assess the performance of the generation models. 200 samples of each model (total 1000 samples) are randomly presented to 3 judges to evaluate the following aspects based on a 2/1/0 score schema: 


\begin{tabular}{lccccc}
\hline Models & $\mathbf{+ 2}$ & $\mathbf{+ 1}$ & $\mathbf{+ 0}$ & Score & Kappa \\
\hline Seq2Seq & $27.1 \%$ & $21.4 \%$ & $51.4 \%$ & 0.756 & 0.4544 \\
Transformer & $42.4 \%$ & $23.6 \%$ & $34.0 \%$ & 1.084 & 0.4429 \\
GPT2 - chitchats & $24.3 \%$ & $19,1 \%$ & $56.6 \%$ & 0.677 & 0.3941 \\
CDialGPT $_{\text {LCCC-base }}$ & $46.7 \%$ & $21.8 \%$ & $31.5 \%$ & 1.152 & 0.3954 \\
CDialGPT $_{\text {LCCC-large }}$ & $\mathbf{4 8 . 6 \%}$ & $\mathbf{2 4 . 5 \%}$ & $\mathbf{2 7 . 0 \%}$ & $\mathbf{1 . 2 1 7}$ & 0.4203 \\
\hline
\end{tabular}

Table 7. Human evaluation.

- Fluency and Relevance If a response is grammatically correct, logically consistent, and relevant to the post, it will get 1 . Otherwise, it will get 0 .

- Informativeness If a response is fluent, relevant, and additionally rich in content, it will get 2 .

The results are shown in Table 7 . We can see that the models trained on LCCC outperform others. Although CDialGPT $\mathrm{LCCC}_{\mathrm{LC} \text {-bese }}$ is not as good as transformer in automatic metrics, CDialGPT $\mathrm{LCCC}_{\mathrm{LC}}$ base performs slightly better than Transformer in manual evaluation. And we computed Fleiss kappa 7 to measure the annotation agreement of crowd workers. The values range within 0.390.44 indicating fair-moderate agreement.

\section{Conclusion}

This paper presents a large-scale cleaned Chinese conversation dataset that is elaborately cleaned by our filtering pipeline. We provide two versions: one is the base version with $6.8 \mathrm{M}$ conversations, and the other is a larger version with $12 \mathrm{M}$ conversations. To obtain high-quality dialogue data, we design rule-based and classifier-based filtering procedures. We also present pre-training models for Chinese dialogue generation, which is trained on the $12 \mathrm{M}$ open-domain conversations. All our pre-training models and the dataset are released for public use.

\section{Acknowledgments}

This work was jointly supported by the National Key R\&D Program of China (Grant No. 2018YFC0830200) and NSFC projects (Key project with No. 61936010 and regular project with No. 61876096). We thank THUNUS NExT Joint-Lab for the support.

\section{References}

1. Adiwardana, D., Luong, M.T., So, D.R., Hall, J., Fiedel, N., Thoppilan, R., Yang, Z., Kulshreshtha, A., Nemade, G., Lu, Y., et al.: Towards a human-like opendomain chatbot. arXiv preprint arXiv:2001.09977 (2020)

2. Bahdanau, D., Cho, K., Bengio, Y.: Neural machine translation by jointly learning to align and translate. arXiv preprint arXiv:1409.0473 (2014) 
3. Bao, S., He, H., Wang, F., Wu, H.: PLATO: pre-trained dialogue generation model with discrete latent variable. CoRR abs/1910.07931 (2019)

4. Cho, K., Van Merriënboer, B., Gulcehre, C., Bahdanau, D., Bougares, F., Schwenk, H., Bengio, Y.: Learning phrase representations using rnn encoder-decoder for statistical machine translation. arXiv preprint arXiv:1406.1078 (2014)

5. Devlin, J., Chang, M.W., Lee, K., Toutanova, K.: Bert: Pre-training of deep bidirectional transformers for language understanding. arXiv preprint arXiv:1810.04805 (2018)

6. Dinan, E., Roller, S., Shuster, K., Fan, A., Auli, M., Weston, J.: Wizard of wikipedia: Knowledge-powered conversational agents. arXiv preprint arXiv:1811.01241 (2018)

7. Fleiss, J.L.: Measuring nominal scale agreement among many raters. Psychological bulletin 76(5), 378 (1971)

8. Holtzman, A., Buys, J., Forbes, M., Choi, Y.: The curious case of neural text degeneration. arXiv preprint arXiv:1904.09751 (2019)

9. Li, C., Wu, Y., Wu, W., Xing, C., Li, Z., Zhou, M.: Detecting context dependent messages in a conversational environment. arXiv preprint arXiv:1611.00483 (2016)

10. Li, J., Galley, M., Brockett, C., Gao, J., Dolan, B.: A diversity-promoting objective function for neural conversation models. arXiv preprint arXiv:1510.03055 (2015)

11. Li, J., Galley, M., Brockett, C., Spithourakis, G.P., Gao, J., Dolan, B.: A personabased neural conversation model. arXiv preprint arXiv:1603.06155 (2016)

12. Li, P.: An empirical investigation of pre-trained transformer language models for open-domain dialogue generation. arXiv preprint arXiv:2003.04195 (2020)

13. Li, Y., Su, H., Shen, X., Li, W., Cao, Z., Niu, S.: Dailydialog: A manually labelled multi-turn dialogue dataset. arXiv preprint arXiv:1710.03957 (2017)

14. Lison, P., Tiedemann, J.: Opensubtitles2016: Extracting large parallel corpora from movie and tv subtitles (2016)

15. Liu, C.W., Lowe, R., Serban, I.V., Noseworthy, M., Charlin, L., Pineau, J.: How not to evaluate your dialogue system: An empirical study of unsupervised evaluation metrics for dialogue response generation. arXiv preprint arXiv:1603.08023 (2016)

16. Loshchilov, I., Hutter, F.: Fixing weight decay regularization in adam (2018)

17. Lowe, R., Pow, N., Serban, I., Pineau, J.: The ubuntu dialogue corpus: A large dataset for research in unstructured multi-turn dialogue systems. arXiv preprint arXiv:1506.08909 (2015)

18. Manning, C.D., Manning, C.D., Schütze, H.: Foundations of statistical natural language processing. MIT press (1999)

19. Mazaré, P.E., Humeau, S., Raison, M., Bordes, A.: Training millions of personalized dialogue agents. arXiv preprint arXiv:1809.01984 (2018)

20. Papineni, K., Roukos, S., Ward, T., Zhu, W.J.: Bleu: a method for automatic evaluation of machine translation. In: Proceedings of the 40th annual meeting on association for computational linguistics. pp. 311-318. Association for Computational Linguistics (2002)

21. Qiu, X., Sun, T., Xu, Y., Shao, Y., Dai, N., Huang, X.: Pre-trained models for natural language processing: A survey. arXiv preprint arXiv:1905.05709 (2020)

22. Radford, A., Narasimhan, K., Salimans, T., Sutskever, I.: Improving language understanding by generative pre-training. URL https://s3-us-west-2. amazonaws. com/openai-assets/researchcovers/languageunsupervised/language understanding paper. pdf (2018)

23. Radford, A., Wu, J., Child, R., Luan, D., Amodei, D., Sutskever, I.: Language models are unsupervised multitask learners. OpenAI Blog 1(8) (2019) 
24. Ritter, A., Cherry, C., Dolan, B.: Unsupervised modeling of twitter conversations. In: Human Language Technologies: The 2010 Annual Conference of the North American Chapter of the Association for Computational Linguistics. pp. 172-180. Association for Computational Linguistics (2010)

25. Rus, V., Lintean, M.: An optimal assessment of natural language student input using word-to-word similarity metrics. In: International Conference on Intelligent Tutoring Systems. pp. 675-676. Springer (2012)

26. Serban, I.V., Sordoni, A., Bengio, Y., Courville, A., Pineau, J.: Hierarchical neural network generative models for movie dialogues. arXiv preprint arXiv:1507.04808 $\mathbf{7}(8)(2015)$

27. Serban, I.V., Lowe, R., Henderson, P., Charlin, L., Pineau, J.: A survey of available corpora for building data-driven dialogue systems. arXiv preprint arXiv:1512.05742 (2015)

28. Shang, L., Lu, Z., Li, H.: Neural responding machine for short-text conversation. arXiv preprint arXiv:1503.02364 (2015)

29. So, D.R., Liang, C., Le, Q.V.: The evolved transformer. arXiv preprint arXiv:1901.11117 (2019)

30. Sordoni, A., Galley, M., Auli, M., Brockett, C., Ji, Y., Mitchell, M., Nie, J.Y., Gao, J., Dolan, B.: A neural network approach to context-sensitive generation of conversational responses. arXiv preprint arXiv:1506.06714 (2015)

31. Sutskever, I., Vinyals, O., Le, Q.V.: Sequence to sequence learning with neural networks. In: Advances in neural information processing systems. pp. 3104-3112 (2014)

32. Vaswani, A., Shazeer, N., Parmar, N., Uszkoreit, J., Jones, L., Gomez, A.N., Kaiser, Ł., Polosukhin, I.: Attention is all you need. In: Advances in neural information processing systems. pp. 5998-6008 (2017)

33. Wang, H., Lu, Z., Li, H., Chen, E.: A dataset for research on short-text conversations. In: Proceedings of the 2013 Conference on Empirical Methods in Natural Language Processing. pp. 935-945 (2013)

34. Wolf, T., Sanh, V., Chaumond, J., Delangue, C.: Transfertransfo: A transfer learning approach for neural network based conversational agents. arXiv preprint arXiv:1901.08149 (2019)

35. Wu, W., Guo, Z., Zhou, X., Wu, H., Zhang, X., Lian, R., Wang, H.: Proactive human-machine conversation with explicit conversation goals. arXiv preprint arXiv:1906.05572 (2019)

36. Wu, Y., Wu, W., Xing, C., Zhou, M., Li, Z.: Sequential matching network: A new architecture for multi-turn response selection in retrieval-based chatbots. arXiv preprint arXiv:1612.01627 (2016)

37. Zhang, S., Dinan, E., Urbanek, J., Szlam, A., Kiela, D., Weston, J.: Personalizing dialogue agents: I have a dog, do you have pets too? arXiv preprint arXiv:1801.07243 (2018)

38. Zhang, Y., Sun, S., Galley, M., Chen, Y.C., Brockett, C., Gao, X., Gao, J., Liu, J., Dolan, B.: Dialogpt: Large-scale generative pre-training for conversational response generation. arXiv preprint arXiv:1911.00536 (2019)

39. Zheng, Y., Chen, G., Huang, M., Liu, S., Zhu, X.: Personalized dialogue generation with diversified traits. arXiv preprint arXiv:1901.09672 (2019)

40. Zhou, K., Prabhumoye, S., Black, A.W.: A dataset for document grounded conversations. arXiv preprint arXiv:1809.07358 (2018) 